\title{
Study on reversal and lateral vibration in the stepped well
}

\author{
Chunjie Han', Ming Guo², Xiangxu Wang ${ }^{3}$, Tie Yan4, Guolin Jing ${ }^{5}$ \\ ${ }^{1,2,3}$ School of Electronic Science, Northeast Petroleum University, Daqing, 163318, China \\ ${ }^{4}$ School of Petroleum Engineering, Northeast Petroleum University, Daqing, 163318, China \\ ${ }^{5}$ School of Chemistry and Chemical Engineering, Northeast Petroleum University, Daqing, 163318, China \\ ${ }^{2}$ Corresponding author \\ E-mail: 1'hcjdqpi@163.com, ${ }^{2} 1154279232 @ q q . c o m,{ }^{3} 1296832933 @ q q . c o m,{ }^{4} y a n t @ e d u . c n$, \\ 5guolinjing@nepu.edu.cn
}

Received 20 September 2019; received in revised form 1 April 2020; accepted 12 June 2020 DOI https://doi.org/10.21595/jve.2020.21046

Check for updates

Copyright (C) 2020 Chunjie Han, et al. This is an open access article distributed under the Creative Commons Attribution License, which permits unrestricted use, distribution, and reproduction in any medium, provided the original work is properly cited.

\begin{abstract}
The reversal and lateral vibration of the drill string are very complex motion and can affect the normal operation of the drill string. The movement of the drill string in the stepped well is different from the movement of the drill string in the regular well. The vibration of the drill string in the stepped well varies with the size of the wellbore and can be visually reflected by the phase speed. To find out the relationship between reversal and lateral vibration, the natural frequency of lateral vibration of the drill string was solved by using the method of energy conservation. The analysis shows that the phase speed of flexural wave in the stepped well is faster in small size wellbore than in large size wellbore. The reversal and lateral resonance is easy to happen in small size wellbore, and the reversal will excite lateral vibration. When the sum of reversal and rotational angular frequencies approaches the natural angular frequency of lateral vibration, the lateral resonance will occur.
\end{abstract}

Keywords: stepped well, drill string, reversal, lateral vibration, phase speed, natural frequency, deflection, bending moment.

\section{Nomenclature}

$d \quad$ Outer diameter of the drill string, $\mathrm{mm}$

$D \quad$ Diameter of the wellbore, mm

$\beta \quad$ Ratio of the diameter of the drill string to the double-sided annulus

$\omega_{p} \quad$ Reversal angular speed of the drill string, $\mathrm{rad} / \mathrm{s}$

$\omega_{r} \quad$ Rotational angular speed of the drill string, $\mathrm{rad} / \mathrm{s}$

$n_{p} \quad$ Reversal speed of the drill string, $\mathrm{r} / \mathrm{min}$

$n_{r} \quad$ Rotational speed of the drill string, $\mathrm{r} / \mathrm{min}$

$R_{F} \quad$ Radius of gyration, $\mathrm{m}$

$V_{a} \quad$ Axial speed of drill string, $\mathrm{m} / \mathrm{s}$

$V_{p} \quad$ Phase speed of flexural wave, $\mathrm{m} / \mathrm{s}$

I

Moment of inertia of the cross-sectional area of the drill string, $\mathrm{m}^{4}$

A Cross-sectional area of the drill string, $\mathrm{m}^{2}$

$m \quad$ Mass of drill string, $\mathrm{kg}$

E Young's modulus of elasticity, $\mathrm{Pa}$

$\omega_{n} \quad$ Natural frequency of drill string, $\mathrm{rad} / \mathrm{s}$

$S \quad$ Tension, $\mathrm{N}$

$x \quad$ Vertical distance from the bottom of the drill string to a point on the drill string, $\mathrm{m}$

$y \quad$ Deformation of drill string at point $x, \mathrm{~m}$

$y_{0} \quad$ Maximum deformation of drill string, $m$

$z \quad$ Vertical distance of drill string, $\mathrm{m}$

$K_{\max } \quad$ Maximum kinetic energy, J 


$\begin{array}{ll}P_{\max } & \text { Maximum potential energy, } \mathrm{J} \\ l & \text { Length of vibration of the drill string, } \mathrm{m} \\ \gamma & \text { Weight per unit volume of drill string, } \mathrm{N} / \mathrm{m}^{3} \\ R_{1} & \text { Single-sided annulus between well wall and joint, } \mathrm{m} \\ L_{1} & \text { Length of single drill string, } \mathrm{m} \\ \omega_{1} & \text { First-order natural frequency of lateral vibration of drill string, rad } / \mathrm{s} \\ g & \text { Gravitational acceleration, } \mathrm{m} / \mathrm{s}^{2} \\ \delta & \text { Deflection of drill string, } \mathrm{m} \\ M & \text { Bending moment of drill string, } \mathrm{N} \cdot \mathrm{m} \\ t & \text { Thickness of drill string, } \mathrm{mm} \\ h & \text { Well depth, mm } \\ \rho_{s} & \text { Density of drill string, } \mathrm{Kg} / \mathrm{m}^{3} \\ \rho_{f} & \text { Density of drilling fluid, } \mathrm{Kg} / \mathrm{m}^{3}\end{array}$

\section{Introduction}

In the process of petroleum drilling, drill string system plays an important role. Appropriate vibration of the drill string is beneficial to clear the bottom cuttings, to improve the rock-breaking efficiency and reduce the sliding friction [1-5]. The reversal and lateral vibration of the drill string in the stepped well is more complicated than that in the regular well. Drill string vibration is one of the most important criteria to measure the performance of drill string system. When the sum of the rotational and reversal angle frequencies of the drill string is close to the lateral vibration natural angular frequency, the lateral resonance will occur $[6,7]$. Collision and pressure will cause serious wear in drill string. To better understand the characteristics of the reversal and lateral vibration in the stepped well and prevent the lateral resonance of the drill string, it is necessary to study the relationship between reversal and lateral vibration.

At present, scholars have made many important achievements in the research of reversal and lateral vibration. Jansen $[8,9]$ studied the lateral vibration of the drill string caused by rotational contact with the wellbore wall, then he designed a control system to reduce lateral vibration, but this system ignored the axial force. Han [10] studied the nature frequency of lateral vibration of the drill string. She concluded that the nature frequency of lateral vibration of the drill string was related to the nature of itself and the axial force exerted. Lian [11] discussed the reversal motion of the drill string and bending frequency, he found that there was not only the rotation but also the reversal in the drill string. The drill string bending frequency was the sum of the drill string rotational speed and the reversal rotational speed. Reversal rotational speed was related to the annulus ratio. Zamani et al. [12] made a review of the drill string failure. They collected and summarized a lot of drill string failure cases, and found that overloading of tension and torque failures accounted for $85 \%$ of total drill string failures. Lin [13] analyzed the failure of oilfield drilling tools showed that most of the failures of drilling tools were due to the reversal and lateral resonance of the drill string. Han and Yan [14] established the lateral vibration equation of drill string in the presence of axial load, and the nature frequency of lateral vibration of the drill string was obtained, and concluded that the reversal of the drill string was an important factor to stimulate the lateral vibration. Tian et al. [15] discussed the effect of lateral inertia on lateral vibration of the drill string. The natural frequency of lateral vibration of the drill string was higher than that without considering the lateral inertia, and the natural frequency gradually decreased with the increasing of drill string length.

The application of stepped wells in practice is very important, but no scholars currently have studied the drill string reversal and lateral vibration in stepped wells. Because of the complexity of reversal and lateral vibration in stepped wells, research on reversal and lateral vibration in stepped wells is necessary. In this paper, the characteristics of reversal of the drill string in the stepped well was researched, and the phase speed was used to illustrate the vibration of the drill 
string in the well. Most scholars selected the infinitesimal of the drill string and established a mechanical equilibrium equation, and substituted boundary conditions into the established equation to obtain the natural frequency of the drill string. In this article, we used the principle of energy conservation, the kinetic energy of the drill string was equal to the portion of the energy converted into potential energy, and established the lateral vibration model of the drill string and solved the natural frequency of lateral vibration of the drill string. By the example analysis of the failures of the drill string, the relationship between the reversal and lateral vibration in the stepped well was found out.

\section{Reversal of drill string}

When the drill string rotates around the axis in the clockwise direction with the rotational speed of the turntable, the joints of the drill string attaches to the well wall due to the centrifugal force. Under the action of the friction between the drill string joint and the well wall, the drill string will rotate around the wellbore axis in the counterclockwise direction along the well wall at a certain speed. It is like the rotational wheel or pipe is rolling forward at a certain speed on the flat ground, but now the ground is replaced by the well wall. This is shown in Fig. 1.

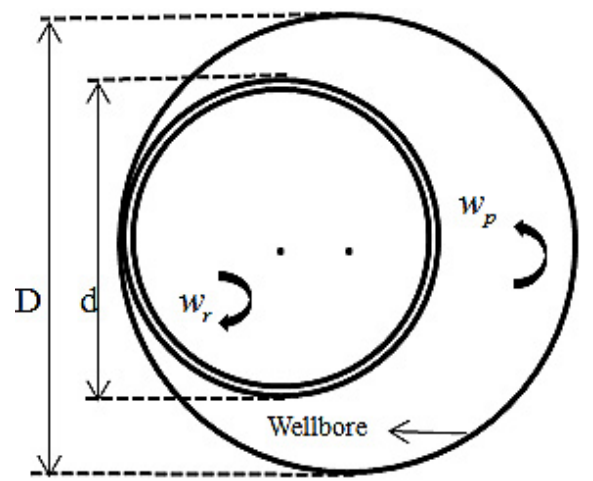

Fig. 1. Diagram of drill string reversal (top view)

When the drill string joint is rolling along the well wall without sliding, the reversal angular speed of the drill string has the following relationship with the angular speed of the turntable.

Annulus ratio can be expressed as:

$\beta=\frac{d}{D-d}$

Relationship between reversal and rotational angular speed of the drill string is:

$\omega_{p}=\beta \omega_{r}$

Relationship between reversal and rotational speed of the drill string is:

$n_{p}=\beta n_{r}$

\subsection{Measured value in stepped well}

The simulated drill string was developed based on theoretical derivation and selection of materials in our university's laboratory. The simulated drill string is a reduced ground drill string model. Wellbore was simulated with transparent polymethyl methacrylate tube [16-18]. The movement of the drill string can be observed and measured by using this simulated drill string 
system on the ground. The geometric similarity ratio between the simulated drill collar and the actual drill collar was $1: 12.55$, and the similarity ratio between the simulated drill string rotational speed and the actual rotational speed was 3.54: 1. By the instrument installed in the model, we measured the reversal trajectory and time of the drill string, and the drill string reversal speed was calculated. We measured the reversal speed of the drill string joint in the stepped well by the manufactured experimental. Fig. 2 shows a model diagram based on measured values.

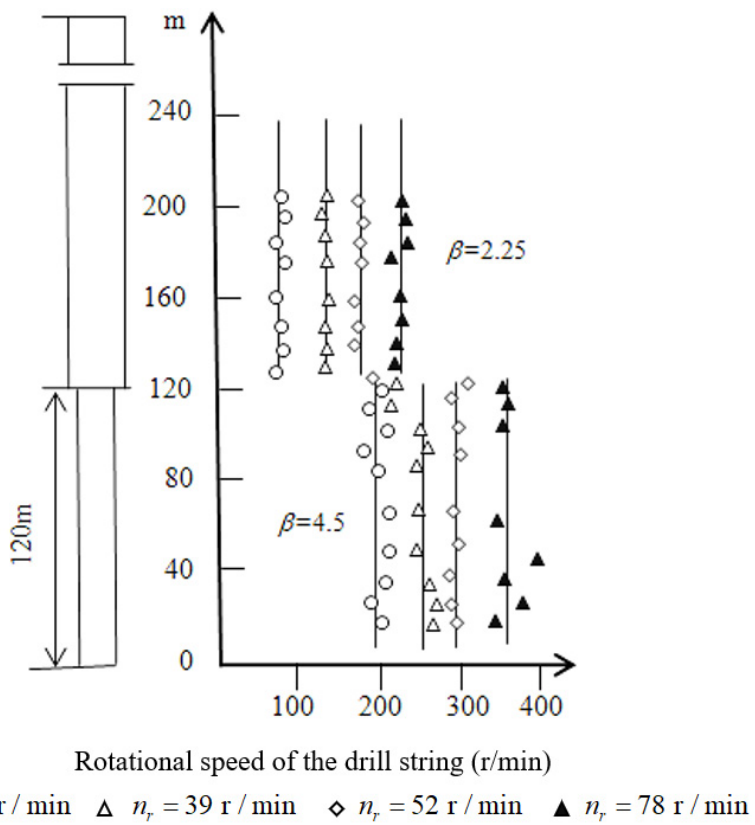

○ $n_{r}=26 \mathrm{r} / \min \Delta n_{r}=39 \mathrm{r} / \mathrm{min} \diamond n_{r}=52 \mathrm{r} / \mathrm{min} \quad \Delta n_{r}=78 \mathrm{r} / \mathrm{min}$

Fig. 2. Reversal rotational speed of joints of drill string in the stepped well

As shown in Fig. 2, the solid lines shows the theoretical values, and the break points shows the measured values of drill string joints. When the value of $\beta$ is small, the measured value is concentrated near the theoretical value. When the value of $\beta$ is large, the measured value is scattered. The reversal rotational speed in the small size well is high, but the reversal rotational speed in the large size well is low. Most of the upper and lower joints of the same drill string has different reversal speed in the same size stepped well. And when the same drill string rotates in two different size stepped wells, rotational speed is different in drill string joints. Especially at 120 meters of stepped well, the upper joint of a drill string is in the large size wellbore and the lower joint is in the small size wellbore. When the turntable speed is $40 \mathrm{r} / \mathrm{min}$, the upper joint reversal speed is $88 \mathrm{r} / \mathrm{min}$, and the lower joint reversal speed is $202 \mathrm{r} / \mathrm{min}$. The difference of reversal speed of the two joints is particularly large. Therefore, when the drill string is drilled in a stepped wellbore and a wellbore with an irregular diameter wellbore, the drill string reversal motion will demonstrate very complicated.

\subsection{Characteristics of reversal in stepped well}

The rotational drill string is usually a complex multi-point self-excited lateral vibration system, so the reversal trajectory of the drill string is generally not circular and no obvious pattern. The relationships between the theoretical value and the measured value of reversal of drill string in stepped well is shown in Fig. 3.

The curve shows the measured value and the straight line shows the theoretical value. The theoretical values 1, 2 and 3 are calculated under the conditions that the values of $\beta$ are 4.5, 2.25 and 0.475 . The reversal motion has a close relationship with the annulus ratio and the rotational 
speed of the drill string, the larger the annulus ratio and the higher the rotational speed of the drill string, the easier it is to reverse [19].

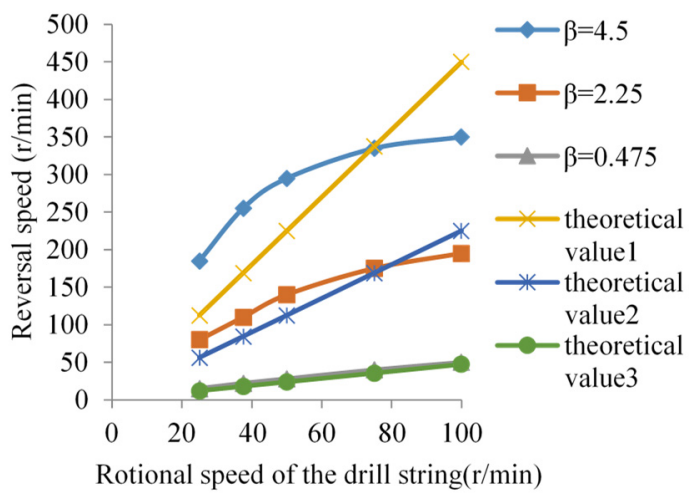

Fig. 3. The relationships between theoretical and the measured value of reversal of the drill string

Fig. 3 shows that the measured value basically does not match the theoretical value. With the annulus ratio increasing, the measured value was deviated from the theoretical value. When the value of $\beta$ is 0.475 , the rotational speed of the drill string is close to the theoretical value. As the value of $\beta$ increasing, the experimental value and the theoretical value begin to deviate. There is a intersection point of the solid line and the broken line, and the reversal rotational speed of the drill string conforms to the measured value at the intersection point.

Combining Fig. 2 with Fig. 3, the measured value before the intersection point is bigger than the theoretical value of reversal, and the measured value is equal to the theoretical value at the intersection point, and the measured reversal speed is less than the drill string speed after the intersection point. This is because when the rotational speed of the drill string is lower than the rotational speed of the drill string at the intersection, the drill string joints does not always keep in contact with the well wall due to the small centrifugal force, and the actual drill string reversal is accompanied by sliding, and often jumps away from the well wall. Therefore, the radius of gyration of the drill string around the axis of the wellbore is shrunk, and the measured value is larger than the theoretical value. At the intersection point, the measured value is equal to the theoretical value, that is because when the drill string contacts with the wellbore and roll, there is the reversal and no sliding at this point. After the intersection point, the drill string joints always keep in contact with the well wall due to the large centrifugal force. Meanwhile, the reversal of the drill string around the wellbore will increase the alternating stress on the drill string, and the higher rotational speed, the greater alternating stress is. Because of unstable centrifugal force, drill string joint knocks the well wall strongly. The impulsive rebound force of the borehole wall on the drill string resists the reversal motion, so the measured value is lower than the theoretical value.

The vibration of the drill string in the stepped well also can be reflected from the phase speed of the flexural wave. If the wave of lateral vibration can be detected, the drill string is in a strong vibration state. The characteristics of flexural wave are different in different sections of stepped well. When the drill string rotates, it looks like a gyro. The radius of gyration of gyro can be expressed as:

$R_{F}=\sqrt{\frac{I}{A}}$.

The axial speed of drill string can be expressed as: 
$V_{a}=\sqrt{\frac{E}{\rho_{s}}}$.

The phase speed of flexural wave can be expressed as:

$V_{p}=\sqrt{R_{F} V_{a} \omega}$

\section{The natural frequency of lateral vibration of drill string}

Assuming that the drill string is under ideal conditions in the following analysis. The speed of the drilling fluid is not considered, and the effects of longitudinal and torsional vibration of drill string on lateral vibration of drill string are neglected. Model of drill string under external excited force is shown in Fig. 4.

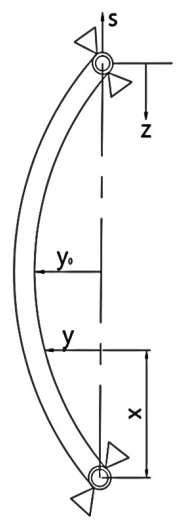

Fig. 4. The bending mechanical model of drill string with external excited force

The relationship between $x$ and $y$ is:

$y=y_{0} \sin n \pi \frac{x}{l}$

$P_{\text {max }}=\frac{1}{2} \int \dot{y}_{\max } d m=\frac{1}{2} \omega^{2} \int y^{2} d m$.

Eq. (8) satisfies the boundary conditions of zero deflection and bending moment at $x=0$ and $x=l$.

Thus:

$P_{\max }=\frac{1}{2} \omega^{2} \int y^{2} d m=\frac{1}{2} \int_{0}^{l} y^{2} \frac{m}{l} d x=\frac{m}{4} \omega^{2} y_{0}^{2}$.

where:

$K_{\text {max }}=\frac{1}{2} \int E I\left(\frac{d^{2} y}{d x^{2}}\right)^{2} d x+z S$,

$\frac{1}{2} \int E I\left(\frac{d^{2} y}{d x^{2}}\right)^{2} d x=\frac{E I}{4} \frac{n^{4} \pi^{4}}{l^{3}} y_{0}{ }^{2}$, 
$z=\int_{0}^{l}\left(\sqrt{\left(1+\left(\frac{d y}{d x}\right)^{2}\right)}-1\right) d x=\int_{0}^{l} \frac{1}{2}\left(\frac{d y}{d x}\right)^{2} d x$

Combining Eq. (11) and Eq. (12), we obtain:

$K_{\max }=\left(\frac{E I}{4} \frac{n^{4} \pi^{4}}{l^{3}}+\frac{S}{4} \frac{n^{2} \pi^{2}}{l}\right) y_{0}{ }^{2}$.

Thus:

$\omega_{n}^{2}=\frac{\left(\frac{E I}{4} \frac{n^{4} \pi^{4}}{l^{3}}+\frac{S}{4} \frac{n^{2} \pi^{2}}{l}\right)}{\frac{m}{4}}$.

Because:

$m=\rho_{s} A l$.

Therefore:

$\omega_{n}=\left(\frac{n \pi}{l}\right)^{2} \sqrt{\frac{E I}{\rho_{s} A}} \sqrt{1+\frac{S}{\left(\frac{n \pi}{l}\right)^{2} E I}}$.

According to Eq. (16), the natural angular frequency of the lateral vibration is related to the nature of the drill string and the axial force exerted.

Eq. (17) and Eq. (18) are for calculating the deflection and bending moment [20]:

$\delta=1.268 \frac{R_{1} \frac{\omega_{p}^{2}}{\omega_{1}^{2}}}{1-\left[\frac{\left(\omega_{p}+\omega_{r}\right)^{2}}{\omega_{1}^{2}}\right]}$

$M=\frac{\gamma A R_{1} \omega_{p}{ }^{2} L_{1}{ }^{2}}{8 g}\left\{1+1.032 \frac{\frac{\left(\omega_{p}+\omega_{r}\right)^{2}}{\omega_{1}{ }^{2}}}{\left|1-\left[\frac{\left(\omega_{p}+\omega_{r}\right)^{2}}{\omega_{1}{ }^{2}}\right]\right|}\right\}$.

From Eq. (17), when the sum of the rotational and reversal angular frequency is greater than the natural angular frequency of the first order, the deflection would be a negative number, and the negative sign means that the direction of vibration is opposite to the positive y-axis. For ease of comparison that all deflection values obtained in the example are taken as absolute values.

\section{Case analysis}

\subsection{Case 1}

With the funding of this project, we carried out on-site inspections of Fuyang No.1 well in Donghai, Shengping No.1 well in Donghe Daqing and 1-H3 double-step wells in Tarim Xinjiang. We found out there was an $86 \%$ agreement by comparing our theoretical research with field data. 
Our theory also guided field experiments, and proved to be extremely available.

Table 1 shows the various parameters of 1-H3 double-step wells in Tarim Xinjiang. Combining Eq. (6) with Eq. (16), the phase speed in the stepped well was calculated, the results are shown in Table 2.

From the calculating results in Table 2, it shows that the phase speed in the stepped well propagates faster in the small size wellbore than that in the large size wellbore. The less the number of the drill string involved in lateral vibration, the faster the phase speed is. This result is caused by the fact that the energy is generated by the vibration of the drill strings. The energy propagates back and forth between the drill string joints, but the energy cannot be released quickly. By detecting the phase speed, the lateral vibration of the drill string in the stepped well can be analyzed. From Case 1, the first-order natural frequency of the lateral vibration in the large size wellbore is smaller than that in small size wellbore, so the drill string is easier to lateral resonance in small size wellbore of the stepped well. By oil file data, it was found that the number of drill strings that failed was due to overloading in the small size wellbore was greater than that in the large size wellbore.

Table 1. Calculating parameters

\begin{tabular}{|c|c|}
\hline Parameter name & Parameter values \\
\hline Diameter of drill string $d /(\mathrm{mm})$ & 89 \\
\hline Thickness of drill string $t /(\mathrm{mm})$ & 9 \\
\hline Well depth $h /(\mathrm{m})$ & 1400 \\
\hline Density of drill string $\rho_{s} /\left(\mathrm{kg} / \mathrm{m}^{3}\right)$ & 7850 \\
\hline Density of drilling fluid $\rho_{f} /\left(\mathrm{kg} / \mathrm{m}^{3}\right)$ & 1200 \\
\hline Gravity acceleration $g /\left(\mathrm{m} / \mathrm{s}^{2}\right)$ & 10 \\
\hline Elastic modulus $E /(\mathrm{GPa})$ & 206 \\
\hline
\end{tabular}

Table 2. Relationships between phase speed and annulus ratio in stepped wells

\begin{tabular}{|c|c|c|c|}
\hline Annulus ratio & Vibration length $(\mathrm{m})$ & Natural frequency $(\mathrm{rad} / \mathrm{s})$ & Phase speed $(\mathrm{m} / \mathrm{s})$ \\
\hline$\beta=2.25$ & 80 & 2.36 & 19 \\
\hline$\beta=2.25$ & 120 & 1.55 & 15.4 \\
\hline$\beta=2.25$ & 160 & 1.14 & 13.2 \\
\hline$\beta=4.5$ & 8 & 21.4 & 57 \\
\hline$\beta=4.5$ & 16 & 10.6 & 40.3 \\
\hline$\beta=4.5$ & 32 & 5.4 & 28.8 \\
\hline
\end{tabular}

\subsection{Case 2}

Table 3 shows the various parameters of the drill string of the Fu Yang No.1 Well in the East China Sea. The deflection and bending moment of the drill string at different rotational speed were analyzed. The relationship between the reversal and the lateral vibration is reflected by the specific data. The results are shown in Table 4.

Table 3. Calculating parameters

\begin{tabular}{|c|c|}
\hline Parameter name & Parameter values \\
\hline Diameter of drill string $d /(\mathrm{mm})$ & 20 \\
\hline Thickness of drill string $t /(\mathrm{mm})$ & 102 \\
\hline Well depth $h /(\mathrm{m})$ & 175 \\
\hline Density of drill string $\rho_{s} /\left(\mathrm{kg} / \mathrm{m}^{3}\right)$ & 7850 \\
\hline Density of drilling fluid $\rho_{f} /\left(\mathrm{kg} / \mathrm{m}^{3}\right)$ & 1200 \\
\hline Gravity acceleration $g /\left(\mathrm{m} / \mathrm{s}^{2}\right)$ & 10 \\
\hline Elastic modulus $E /(\mathrm{GPa})$ & 206 \\
\hline
\end{tabular}


Table 4. Puncture points of different wells and their natural frequencies

\begin{tabular}{|c|c|c|c|}
\hline Serial number & Well depth (m) & $\begin{array}{c}\text { Distance from the point of the } \\
\text { puncture to the wellhead (m) }\end{array}$ & Natural frequency (rad/s) \\
\hline 1 & 3271 & 145 & 24.7 \\
\hline 2 & 3357 & 147 & 25.04 \\
\hline 3 & 3780 & 125 & 26.8 \\
\hline 4 & 4360 & 136 & 31.3 \\
\hline
\end{tabular}

Fig. 5 shows the deflection and bending moment value in different rotational speed of drill string.

From Fig. 5, when the rotational speed is $110 \mathrm{r} / \mathrm{min}$, the values of deflection and bending moment of the No. 3 are 5 to 10 times of that of the other three groups. Combine Eq. (17), it can be concluded that the reversal will induce lateral vibration of the drill string. When the rotational speed of drill string is $110 \mathrm{r} / \mathrm{min}$, the sum of the rotational and reversal angular frequencies of the drill string is $26.4 \mathrm{rad} / \mathrm{s}$, and the natural frequency of this point is $26.8 \mathrm{rad} / \mathrm{s}$, so the drill string is nearly in lateral resonance. The single-sided annulus between the well wall and the joint is $0.0255 \mathrm{~m}$, but the deflection value in the resonance is $0.620 \mathrm{~m}$, so the drill string will have strong collision with the well wall, and the drill string will be subjected to the extremely strong reaction. Large bending stresses may cause puncture and other failures in the drill string in a short period of time.

In Case 2, the value of $\beta$ is 1.4. And the rotational speed of drill string are $100 \mathrm{r} / \mathrm{min}, 110 \mathrm{r} / \mathrm{min}$ and $120 \mathrm{r} / \mathrm{min}$, so the sums of the rotational and reversal angular frequencies of the drill string in these three-speed are $24 \mathrm{rad} / \mathrm{s}, 26.4 \mathrm{rad} / \mathrm{s}$ and $28.8 \mathrm{rad} / \mathrm{s}$. Combining Table 4 with Fig. 5, the closer the sum of the rotational and reversal angular frequencies to the natural frequency of lateral vibration of the drill string, the easier it is to stimulate lateral resonance in the drill string.

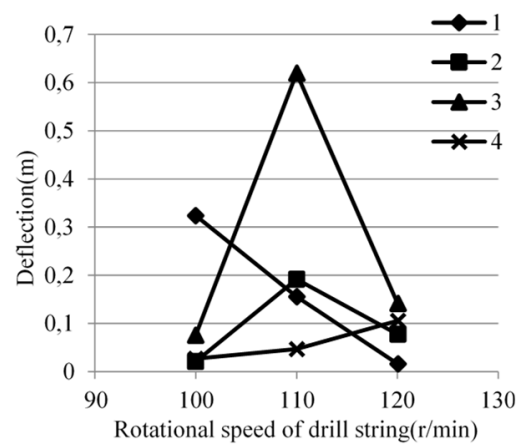

a)

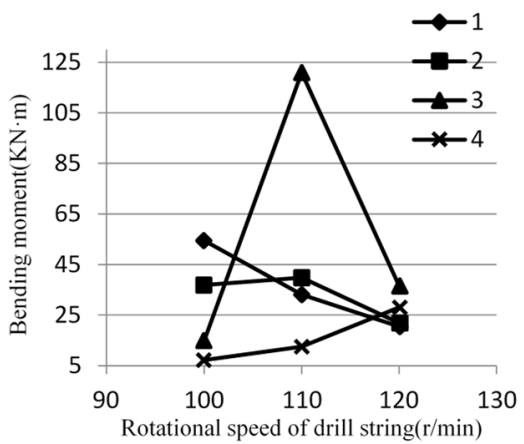

b)

Fig. 5. Deflection and bending moment of the puncture point at different speeds

\section{Conclusions}

1) Reversal of drill string at different sections in a stepped well is different. When the annulus ratio is small, the theoretical value and the measured value basically match. With the annulus rate increasing, the measured value begins to deviate the theoretical value.

2) From the phase speed, it can be seen that the phase speed of well sections with small annulus rate is small, and the phase speed of well sections with large annulus rate is large. The phase speed of flexural wave in the stepped well is faster in small size wellbore than in large size wellbore. The reversal and lateral resonance is easy to happen in small size wellbore.

3) The reversal will stimulate the lateral vibration of the drill string. The closer the sum of the drill string reversal and the rotational angle frequency to the natural angular frequency of the drill string, the easier it is to stimulate lateral resonance. Deflection and bending moment are important quantities to measure drill string failure. The lateral resonance of the drill string will cause serious 
damage in a short time, and the rotational speed of drill string should be adjusted, so that the sum of the drill string reversal and the rotational angle frequency is far from the natural angular frequency of lateral vibration of the drill string.

\section{Acknowledgements}

This work is supported by the National Major Special Project that Drilling Engineering Integration Software (No. 2016ZX05020-006). Great thanks to my teachers and classmates for their help during the writing process.

\section{References}

[1] Wang Zhigang, Liang Jian, Liu Xiumei Analysis of failure behavior of drill string in deep geological core drilling. Exploration Engineering (Rock Soil Drilling and Tunneling), Vol. 45, Issue 12, 2018, p. 28-31, (in Chinese).

[2] Wang Xiyou, Wang Chang, Xiong Jiyou, Xue chenggang, Long Gang Failure analysis of drilling tool leakage in tarim Oil field in 2005. Henan Petroleum, Vol. 1, 2006, p. 69-70, (in Chinese).

[3] Chen Yaohui, Bi Xueliang, Yan Tie Analysis on drill string vibration of deep wells. Natural Gas Industry, Vol. 25, Issue 9, 2005, p. 76-79, (in Chinese).

[4] Lan Kai, Liu Xiangfeng Research advances in drill string excessive vibration identification and control techniques. Journal of Jilin University (Geoscience Edition), Vol. 47, Issue 1, 2017, p. 203-214, (in Chinese).

[5] Yang Jian-bo, Jiang Ping Study on fatigue life prediction of air drilling drill String. West-China Exploration Engineering, Vol. 21, Issue 4, 2009, p. 61-66, (in Chinese).

[6] Yang C., Wang R., Han L., Xue Q. Analysis on the lateral vibration of drill string by mass effect of drilling fluid. Journal Mechanical Sciences, Vol. 10, 2019, p. 363-371.

[7] Li Dong-yang, Chen Cicang, Wang Qianqian An experimental study of drill string failure in gas drilling. China Petroleum Machinery, Vol. 40, Issue 7, 2012, p. 29-33, (in Chinese).

[8] Jansen J. D., Van den Steen L. Active damping of self-excited torsional vibration in oil well drillstrings. Journal of Sound and Vibration, Vol. 179, Issue 4, 1995, p. 647-668.

[9] Jansen J. D. Non-linear rotor dynamic as applied to oil well drill string vibrations. Journal of Sound and Vibration, Vol. 147, Issue 1, 1991, p. 115-135.

[10] Han Chunjie, Yan Tie, Bi Xueliang Rules and their application of drilling string lateral vibration. Journal of Daqing Petroleum Institute, Vol. 1, 2004, p. 14-16, (in Chinese).

[11] Lian Zhanghua, Luo FaQian, Liu Yuanyang Drill string reversal motion and bending frequency analysis. Journal of Southwest Petroleum Institute, Vol. 3, 2004, p. 75-78, (in Chinese).

[12] Zamani S. M., Hassanzadeh Tabrizi S.-A., Sharifi H. Failure analysis of drill string: A review. Engineering Failure Analysis, Vol. 59, 2016, p. 605-623.

[13] Lin Yuanhua, Luo Faqian, Shi Taihe Reasons for failure of drilling tools in Tarim Oilfield. Drilling Production Technology, Vol. 5, 2007, p. 21-23, (in Chinese).

[14] Han Chunjie, Yan Tie Study on the law of lateral vibration of drill string under reversal circumstance. Drilling and Production Technology, Vol. 1, 2007, p. 80-83, (in Chinese).

[15] Tian Jialin, Wu Chunming, Yang Lin Study on lateral vibration characteristics of a drill string with lateral inertia effect. Journal of Failure Analysis and Prevention, Vol. 16, Issue 4, 2016, p. 678-682.

[16] Gee R., Hanley C., Hussain R., Canuel L., Martinez J. Axial oscillation tool vs. lateral vibration tools for friction reduction-what's the best way to shake the pipe. SPE/IADC Drilling Conference and Exhibition, London, England, 2015.

[17] Altamimi Ibrahim M., Mokrani Samir, Zulkaf Ahmed H. Axial oscillation tool significantly mitigates the vibration level and enhances drilling performance in conjunction with standard RSS system. Abu Dhabi International Petroleum Exhibition and Conference, 2015.

[18] Jones Steve, Feddema Chad, Sugiura Junichi, Lightey Jeff A new friction reduction tool with axial oscillation increases drilling performance: field-testing with multiple vibration sensors in one drill string. IADC/SPE Drilling Conference and Exhibition, Fort Worth, Texas, 2016.

[19] Zhang Yanglie Kinematics and Dynamics of Drill String. Beijing, 2000, (in Chinese). 


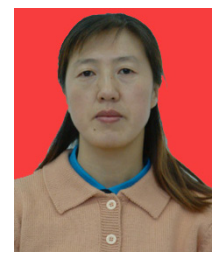

Chunjie Han received Ph.D. degree in School of Petroleum Engineering from Northeast Petroleum university, Daqing, China, in 2010. Now she works at Northeast Petroleum University, Daqing. Her current research interests include drill string mechanics and drilling engineering.

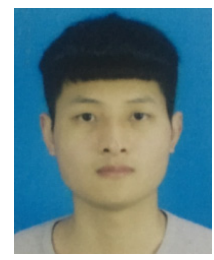

Ming Guo is a student in School of Electronic Science and Northeast Petroleum University, Daqing. Now he is engaged in drill string mechanics.

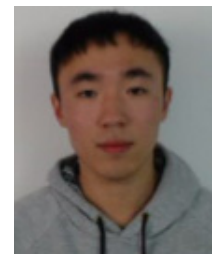

Xiangxu Wang is a student in School of Electronic Science and Northeast Petroleum University, Daqing. Now he is engaged in drilling engineering shock absorber.

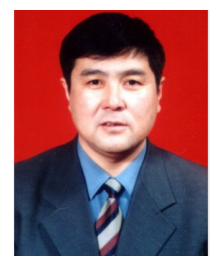

Tie Yan received Ph.D. degree in School Civil Engineering from Harin Institute of Technology, Harbin, China, in 2010. Now he works at Northeast Petroleum University, Daqing. His current research interests include drilling engineering, oil and gas field development and exploitation engineering oil and gas field construction project.

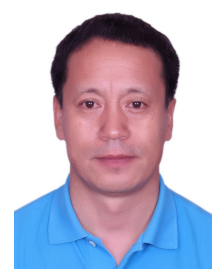

Guolin Jing received Ph.D. degree in School of Environmental Engineering from Harbin Institute of Technology, Harbin, China, 2004. Now he works at Northeast Petroleum University, Daqing. His current research interest includes the harmless technology research of supercritical waste water. 